den Schluß zu, daß bei diesen Temperaturen eine elektrolytische Komponente der Leitfähigkeit vorhanden sein muß. Es setzt eine Wanderung des Schwefels ein, die sich bis zur Absetzung des Schwefels an der Anode steigern läßt. Die Leitfähigkeitsmessungen bis $\mathrm{zu} \sim 300^{\circ} \mathrm{C}$ stimmen mit den Ergebnissen von $\mathrm{Hin}$ te $\mathrm{n}$ berger im wesentlichen überein.

Es drängt sich bei diesen Ergebnissen und den daraus gezogenen Folgerungen die Frage auf, ob auch der Sperrschichteffekt am PbSeine chemische Reaktion zwischen Sauerstoff und Bleisulfid fordert. Hochempfindliche PbS-Sperrschichtzellen herzustellen, ist unseres Wissens noch nicht gelungen. Der v́on uns bisher erzielte Effekt liegt schätzungsweise noch um zwei Größenordnungen unter dem, der sich erzielen lassen müßte. Wir können daher über obige Frage noch nicht diskutieren. Hingegen liegen einige Erfahrungen an natürlichen PbS-Kristallen vor. Bekanntlich kann man aus natürlichem PbS-Kristall eine Zelle herstellen, die eine selbständige EMK bei Belichtung liefert ${ }^{5}$ (genannt Bleiglanz-Netzzelle). Man poliert einen Kristall und drückt von der Lichteinfallseite aus ein feinmaschiges Drahtnetz auf. Bemerkenswert ist, daß Einkristalle und grobkristal-

5 Nicht öffentlicher Vortrag von P. Görlich u. K. G r o $B \mathrm{ku} \mathrm{r} \mathrm{th} \mathrm{in} \mathrm{,Forschungsarbeiten} \mathrm{über} \mathrm{infra-}$ rote Strahlungsempfänger“, Berlin 1944. line Stücke keinen Effekt geben. Lediglich Kristalle mit feinkristallinem Gefüge bestimmter Fundorte (z. B. Sardinien) sind zur Herstellung von Netzzellen geeignet. Zunächst scheint die mit den PbS-Widerstandszellen übereinstimmende spektrale Verteilung der PbS-Netzzellen darauf hinzuweisen, daß die gleiche Gitteranordnung wie bei den PbS-Widerstandszellen als Ursprungsort der ausgelösten Elektronen zu gelten hat. Es fragt sich dann, wo die Möglichkeit zu einer chemischen Reaktion zwischen Sauerstoff und Bleisulfid im natürlichen $\mathrm{PbS}$ bestehen konnte. Vermutlich hat man hierzu die Oberfläche der feinen Kristallite in Betracht zu ziehen. Dagegen dürfte die Entstehung eines grobkristallinen Gefüges bzw. eines Einkristalles unter Bedingungen geschehen sein, die eine chemische Reaktion zwischen Sauerstoff und Bleisulfid an den Grenzen der Kristalle bzw. des Einkristalles nicht zuließen (fehlende hohe Temperatur und fehlender hoher Druck bei der Entstehung der Kristalle, also Wachstum ohne äußere Hemmungen).

Wie weit es uns möglich ist, in Zukunft quantitative Messungen zur vollständigen Aufklärung der Erscheinungen durchzuführen, läßt sich im Augenblick noch nicht übersehen, so daß wir uns vorläufig mit den vorstehenden kurzen Angaben begnügen müssen.

\title{
Die Abscheidung strömender Apiezonölnebel durch Thermodiffusion
}

\author{
Von Richard Weber* \\ (Z. Naturforschg. 2 a, 48-55 [1947]; eingegangen am 5. Okt. 1946)
}

\begin{abstract}
Es wird eine apparative Anordnung angegeben, mit deren Hilfe hochverdünnte Apiezonölnebel in reproduzierbarer Weise erzeugt werden können. Um die Konzentration dieser Nebel zu verfolgen, werden dieselben unter dem Tyndallometer beobachtet und in konstanten Zeitabständen von 2 min die Streulichtintensität gemessen. Hierbei bleibt die Streulichtintensität während einer Versuchsdauer von 1 Stde. konstant. - Anschließend wird die Abscheidung der Nebel beim Durchgang durch ein Thermodiffusionsrohr studiert. Hierbei gelingt es, die Abscheidung in Abhängigkeit von Temperaturgradient, Strömungsgeschwin digkeit und Massenkonzentration quantitativ zu erfassen. Für kleine Strömungsgeschwindigkeiten stimmt die im thermischen Felde resultierende Geschwindigkeit der Teilchen mit der an Hand einer Gleichung von A. Einstein ermittelten überein.
\end{abstract}

$\mathrm{I}^{\mathrm{m}}$ m Rahmen einer Reihe von Arbeiten über die Isotopentrennung durch Thermodiffusion wird darauf hingewiesen, daß sich der Thermodiffu-

* z. Zt. Dickschied über Bad Schwalbach (Taunus). Die Versuche zu dieser Arbeit wurden im Laboratorium sionseffekt besonders eindrucksvoll an Aerosolteilchen demonstrieren läßt, wenn man das von Clusius und Dickel angegebene Trennrohr zu der Auer-Gesellschaft, Werk Oranienburg bei Berlin, durchgeführt. 
Hilfe nimmt ${ }^{1}$. Aerosolteilchen, die dem Temperaturgefälle eines solchen Trennrohres ausgesetzt werden, wandern wegen ihres großen Masseunterschiedes gegenüber dem Trägergas in Richtung auf die kältere Rohrwand hin und werden, falls der Temperaturgradient genügend groß gewählt wird, dort abgeschieden.

Über die Abscheidung von Aerosolteilchen an gegenüber ihrer Umgebung kälteren Oberflächen durch Thermodiffusion liegt auch noch anderes Beobachtungsmaterial vor ${ }^{2}$. Eine Reihe von Forschern hat auch versucht, das Zustandekommen des Thermodiffusionseffektes an Schwebeteilchen zu erklären, und hat entsprechende Rechnungen angestellt ${ }^{3}$. Ausreichendes Versuchsmaterial fehlt aber noch ganz. Dabei sind solche Versuche nicht nur aus theoretischen, sondern auch aus praktischen Gründen von Bedeutung ${ }^{4}$. Als Ziel der vorliegenden Arbeit wurde deshalb angesehen, die Abscheidung eines Aerosols beim Strömen durch ein Rohr mit axialem Heizdraht als Funktion des Temperaturgradienten, des Strömungsvolumens und der Aerosolkonzentration festzulegen.

Die Durchführung der Versuche hing von der Erzeugung eines Aerosols ab, das folgende Anforderungen in möglichst weitgehendem Maße erfüllen mußte:

1. Es mußte sich bezüglich Teilchenzahl pro Volumeneinheit. und Massenkonzentration in reproduzierbarer Weise darstellen lassen.

2. Die im Aerosol sich abspielenden zeitlichen Veränderungen infolge Koagulation der einzelnen Teilchen durften nur äußerst gering sein. Somit konnte also auch nur ein Aerosol mit sehr geringer Massenkonzentration in Frage kommen.

3. Verdampfungserscheinungen an den einzelnen Aerosolteilchen durften nicht auftreten.

Im Hinblick auf Punkt 3 schien somit ein Aerosol mit fester disperser Phase am geeignetsten. Es

1 K. Clusius u. G. Dickel, Z. physik. Chem. (Abt. B) 44, 445 [1939]; H. Jensen, Angew. Chem. 54, 407 [1941].

2 T. L. I b b s, Trans. Faraday Soc. 32, 1082 [1936]; K. Clusius, Z. Ver. dtsch. Ing., Beiheft Verfahrenstechnik Nr. 2, S. 23 [1941].

${ }^{3}$ A. Einstein, Z. Physik 27, 1 [1924]; W. Caw o od, Trans. Faraday Soc. 32, 1068 [1936]; K. Cl u sius, Z. Ver. dtsch. Ing., Beiheft Verfahrenstechnik Nr. 2, S. 23 [1941].

* Vergl. deutsche Patentanmeldung A 94394 IVb/12e des Verf. vom 28. Okt. 1941. geht jedoch aus den bekannten Arbeiten über Aerosole mit fester disperser Phase hervor, daß die Punkte 1 und 2 an keinem derselben in einfacher Weise $\mathrm{zu}$ verwirklichen sind. Punkt 2 vor allem deswegen nicht, da fast sämtliche untersuchten Systeme mit fester disperser Phase strukturierte Teilchen aufweisen, wodurch häufig die Koagulation in starkem Maße begünstigt wird ${ }^{5}$. Da sich die Punkte 1 und 2 mit Hilfe eines Zerstäubernebels und öl als Zerstäuberflüssigkeit weitgehend verwirklichen ließen, wurde schließlich versucht, mit einem solchen Ölnebel zum Ziele $\mathrm{zu}$ gelangen. Im Hinblick auf Punkt 3 wurde Apiezonöl als Zerstäuberflüssigkeit verwendet. Dieses Öl besitzt nämlich einen ungewöhnlich niedrigen Dampfdruck, der bei gew öhnlicher Temperatur größenordnungsmäßig bei $1 \times 10^{-7} \mathrm{~mm} \mathrm{Hg}$ $\operatorname{liegt^{6}}$.

\section{Beschreibung der Versuchs- anordnung}

Die Versuchsanordnung ist in Abb.1 wiedergegeben und setzte sich aus folgenden Teilen zusammen: dem Trennrohr $T r$ aus Glas, in dem die Abscheidung der strömenden Ölnebelteilchen vorgenommen wurde, einer Zerstäubervorrichtung $Z$ mit Spirale nach Regener ${ }^{7}$, die sich in einem auf $30^{\circ} \mathrm{C}$ eingestellten Ultrathermostaten eingebaut befand und über einen Dreiweghahn $D$ mit dem $2000 l$ fassenden Gasraum $G$ leitend verbunden werden konnte. Der Gasraum bestand aus einer Stahlgerüstkonstruktion mit eingekitteten Glasscheiben. Mit dem unteren und oberen Rohrende war das Trennrohr durch weite Glasrohre und Nebelschläuche an den Gasraum bzw. das Tyndallometer $T y^{8}$ angeschlossen.

Hinter dem Tyndallometer war ein Schwebstoffilter $S$ angebracht, damit der Luftstrom von den mitgeführten Ölnebelteilchen befreit und die Strömungsgeschwindigkeit desselben anschließend mit Hilfe eines Rotamessers $R$ eingestellt werden konnte. Über ein Reduzierventil $\operatorname{Red}_{2}$ war die Versuchsapparatur an ein Vakuum angeschlossen.

Das Trennrohr war $1 \mathrm{~m}$ lang und hatte eine $1 . \mathrm{W}$. von $13 \mathrm{~mm}$. Es war von einem gläsernen Kühler umgeben, der mit Leitungswasser von einer mittleren Temperatur von $23^{\circ}$ gespeist wurde. Als Heizdraht wurde ein Cr-Ni-Draht von 0,3 mm Stärke verwendet.

5 G. W i e g n e r, Kolloid-Z. 58, 157 [1932]; D. B e is ch e r u. A. W i n k e l, Naturwiss. 25, 420 [1937].

${ }_{6}^{6}$ H. K l u m b, Naturwiss. 19, 612 [1931].

7 H. Freundilch, Kapillarchemie II. Bd., S. 787 [1932].

8 Bei dem benutzten Tyndallometer handelte es sich um ein Gerät der Firma Leitz, Wetzlar; Literatur: M. B e r ek, K. Männ ch en u. W. S chäf er, Z. Instrumentenkunde 56, 49 [1936]. 


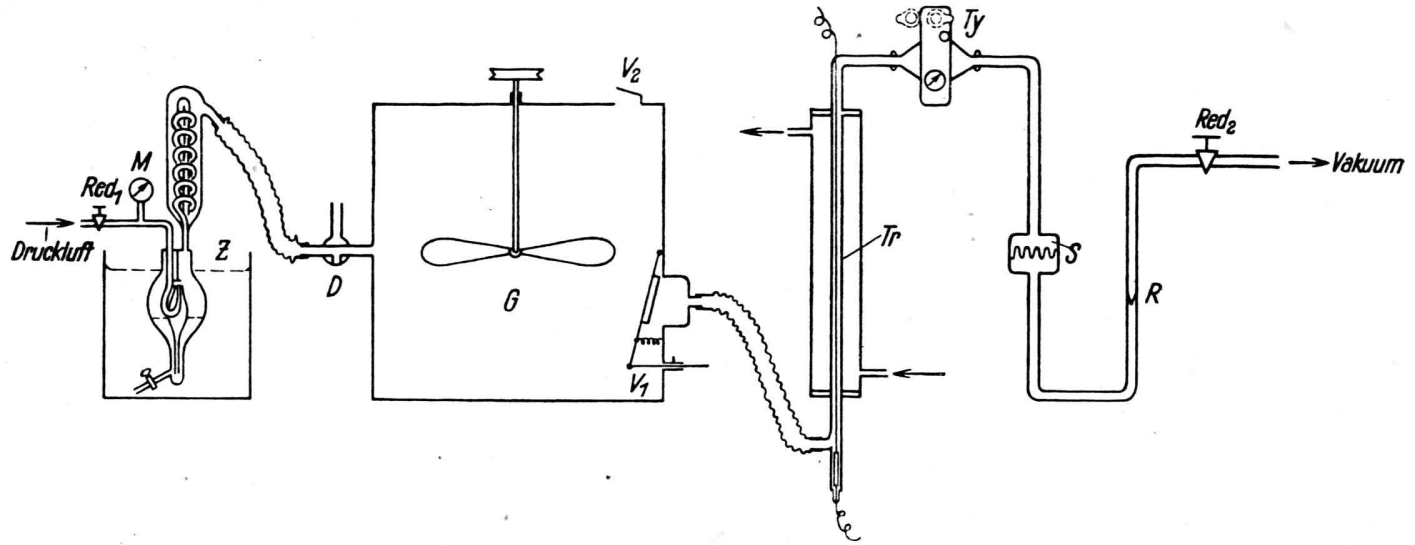

Abb. 1.

Tr Trennrohr

Z Zerstäubervorrichtung mit Manometer $M$

D Dreiweghahn
G Gasraum

Ty Tyndallometer

S Schwebstoffilter
R Rotamesser

Red Reduzierventile

$\hat{\nabla}$ Gasraumverschlüsse
Dieser befand sich oben an einem eingeschmolzenen Platindraht angelötet und war an seinem unteren Ende durch ein stählernes Gewicht $G e$ beschwert, das mit einem angelöteten Platindrahtfortsatz versehen war. Letzterer tauchte in Quecksilber, das die Stromzufuhr besorgte. Eine gute Zentrierung des Cr-NiDrahtes wurde durch je eine oben und unten inmitten des Trennrohres eingeschmolzene Glasöse erreicht.

\section{Die tyndallometrische Konzentra-} tionsbestimmung der Ölnebel (Eichung des Tyndallometers)

Die Teilchenkonzentration eines Aerosols wird am genauesten durch Auszählen der Teilchen im Ultramikroskop ermittelt. Diese Methode konnte jedoch nicht herangezogen werden, da der Vorgang des Auszählens zu viel Zeit beansprucht, andererseits aber plötzlich hervorgerufene Veränderungen in der Teilchenkonzentration der ölnebel schnell erkannt werden mußten (vergl. die Versuche in Abschn. III). Hierzu schien das tyndallometrische Verfahren besonders geeignet. Dieses Verfahren kann jedoch nur bei kleinen Aerosolkonzentrationen angewendet werden, da hier die Teilchenzahl direkt proportional der Intensität des von den Teilchen gestreuten Lichtes ist ${ }^{9}$.

Die Herstellung der Nebel geschah in dem 2000-l-Gasraum. Je nach dem gewünschten Verdünnungsgrad wurde der Zerstäubernebel eine bestimmte Zeit lang eingeblasen und mittels einer langsam laufenden Luftschraube über das ganze

9 H. Sa u e r, Z. Instrumentenkunde 51, 408 [1931]; R. C. Tolm an, L. H. Rey er son, E. B. Vliet, R. H. Gerke u. A. P. Brooks, J. Amer. chem. Soc. 41, 300 [1919].

10 Aus räumlichen Gründen kann hier wie auch später in Tab. 2 nur ein kleiner Teil des Versuchsmaterials wiedergegeben werden.
Gasraumvolumen gleichmäßig verteilt. Auf diese Weise wurden auch die verschiedenen Nebelkonzentrationen für die Eichung des Tyndallometers eingestellt. Die Einleitungszeiten des Zerstäubernebels betrugen zwischen 40 und 6 sec, was bei einer Strömungsgeschwindigkeit des Zerstäubernebels von $25 \mathrm{l} / \mathrm{min}$ einer $167: 800$-fachen Verdünnung entsprach. Für diese Versuche wurde das Trennrohr ausgebaut und das Tyndallometer un mittelbar an den Gasraum angeschlossen. Ein Versuch ging in der Weise vonstatten, daß der im Gasraum verdünnte Zerstäubernebel mit konstanter Strömungsgeschwindigkeit aus dem Gasraum durch das Tyndallometer abgesaugt und in konstanten Zeitabständen die Intensität des an den Nebelteilchen gestreuten Lichtes fortlaufend gemessen wurde. Diese Versuche hatten gleichzeitig den Sinn, ein Bild über die zeitlichen Veränderungen an den Nebelsystemen zu gewinnen. Deshalb wurde bei einigen Meßreihen die Versuchsdauer auf etwa $30 \mathrm{bzw}$. 60 min ausgedehnt. Einige dieser Versuchsreihen werden in Tab. 1 mitgeteilt ${ }^{10}$. In denselben bedeutet $T$ die Einleitungszeit des Zerstäubernebels in den Gasraum, $c$ die der Einleitungszeit entsprechende Konzentration ${ }^{11}$ des Gas-

11 Die Massenkonzentration des Gasraumnebels wurde aus dem Verdünnungsgrad und der bekannten Konzentration des Zerstäubernebels errechnet. Zur Ermittlung der Konzentration des Zerstäubernebels in $\mathrm{mg} / \mathrm{m}^{3}$ wurde derselbe unter dem normalen Betriebsdruck von 2.5 Atm. aus dem Zerstäuber abgeblasen. Hierbei betrug die Strömungsgeschwindigkeit des abgeblasenen Nebels $25 \mathrm{l} / \mathrm{min}$. Die nebelbeladene Luft wurde 10 oder 15 min durch zwei hintereinander geschaltete Cemopapierfilter geleitet, die also die in einem Volumen 
Vers. Nr. $12 T=6 \mathrm{sec}, c=5,1 \mathrm{mg} O ̈ \mathrm{l} / \mathrm{m}^{3}, v=3 \mathrm{l} / \mathrm{min}$.

\begin{tabular}{|c|c|c|c|c|c|c|}
\hline $\begin{array}{ll}t & 3^{\prime} 6^{\prime \prime} \\
I & 0,484\end{array}$ & $\begin{array}{c}5^{\prime} 6^{\prime \prime} \\
0,509\end{array}$ & $\begin{array}{l}87^{\prime} 6^{\prime \prime} \\
0,505\end{array}$ & $\begin{array}{c}9^{\prime} 6^{\prime \prime} \\
0,495\end{array}$ & $\begin{array}{l}11^{\prime} 6^{\prime \prime} \\
0,502\end{array}$ & $\begin{array}{l}13^{\prime} 6^{\prime \prime} \\
0,502\end{array}$ & $\begin{array}{l}15^{\prime} 6^{\prime \prime} \\
0,512\end{array}$ \\
\hline $\begin{array}{ll}t & 17^{\prime} 6^{\prime \prime} \\
I & 0,516\end{array}$ & $\begin{array}{l}20^{\prime} 6^{\prime \prime} \\
0,509\end{array}$ & $\begin{array}{l}22^{\prime} 6^{\prime \prime} \\
0,507\end{array}$ & $\begin{array}{l}24^{\prime} 6^{\prime \prime} \\
0,507\end{array}$ & $\begin{array}{l}26^{\prime} 6^{\prime \prime} \\
0,509\end{array}$ & $\begin{array}{l}28^{\prime} 6^{\prime \prime} \\
0,502\end{array}$ & $\begin{array}{l}30^{\prime} 6^{\prime \prime} \\
0,505\end{array}$ \\
\hline 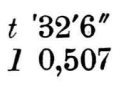 & $\begin{array}{l}34^{\prime} 6^{\prime \prime} \\
0,495\end{array}$ & $\begin{array}{l}36^{\prime} 6^{\prime \prime} \\
0,484\end{array}$ & $\begin{array}{l}38^{\prime} 6^{\prime \prime} \\
0,490\end{array}$ & $\begin{array}{l}40^{\prime} 6^{\prime \prime} \\
0,491\end{array}$ & $\begin{array}{l}42^{\prime} 6^{\prime \prime} \\
0,493\end{array}$ & $\begin{array}{l}44^{\prime} 6^{\prime \prime} \\
0,495\end{array}$ \\
\hline $\begin{array}{ll}t & 46^{\prime} 6^{\prime \prime} \\
I & 0,486\end{array}$ & $\begin{array}{l}48^{\prime} 6^{\prime \prime} \\
0,488\end{array}$ & $\begin{array}{l}50^{\prime} 6^{\prime \prime} \\
0,488\end{array}$ & $\begin{array}{l}52^{\prime} 6^{\prime \prime} \\
0,493\end{array}$ & $\begin{array}{l}54^{\prime} 6^{\prime \prime} \\
0,493\end{array}$ & $\begin{array}{l}56^{\prime} 6^{\prime \prime} \\
0,479\end{array}$ & $\begin{array}{l}58^{\prime} 6^{\prime \prime} \\
0,472\end{array}$ \\
\hline $\begin{array}{l}t \quad 60^{\prime} 6^{\prime \prime} \\
I \quad 0,476\end{array}$ & $\begin{array}{l}62^{\prime} 6^{\prime \prime} \\
0,477\end{array}$ & $\begin{array}{l}64^{\prime} 6^{\prime \prime} \\
0,472\end{array}$ & & & & \\
\hline
\end{tabular}

Vers. Nr. $14 T=6 \mathrm{sec}, c=5,1 \mathrm{mg}$ Öl $/ \mathrm{m}^{3}, v=3 \mathrm{l} / \mathrm{min}$.

$t \quad 3^{\prime} 6^{\prime \prime} \quad 11^{\prime} 6^{\prime \prime} \quad 19^{\prime} 6^{\prime \prime} \quad 27^{\prime} 6^{\prime \prime} \quad 35^{\prime} 6^{\prime \prime} \quad 43^{\prime} 6^{\prime \prime} \quad 51^{\prime} 6^{\prime \prime}$

$\begin{array}{llllllll}I & 0,505 & 0,495 & 0,504 & 0,495 & 0,502 & 0,488 & 0,479\end{array}$

$t 59^{\prime} 6^{\prime \prime}$

I 0,472

Vers. Nr. $6 T=11 \mathrm{sec}, c=9,3 \mathrm{mg} \ddot{\mathrm{Ol}} / \mathrm{m}^{3}, v=3 \mathrm{l} / \mathrm{min}$.

$t 4^{\prime} 11^{\prime \prime} 12^{\prime} 11^{\prime \prime}, 20^{\prime} 11^{\prime \prime} \quad 28^{\prime} 11^{\prime \prime} 36^{\prime} 11^{\prime \prime} 44^{\prime} 11^{\prime \prime}$

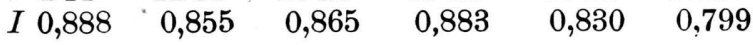

$t 54^{\prime} 11^{\prime \prime} 60^{\prime} 11^{\prime \prime}$

$\begin{array}{lll}I & 0,806 & 0,802\end{array}$

Vers. Nr. $9 \quad T=11 \mathrm{sec}, c=9,3 \mathrm{mg}$ Öl $/ \mathrm{m}^{3}, v=3 \mathrm{l} / \mathrm{min}$.

$\begin{array}{llccccc}t & 7^{\prime} 11^{\prime \prime} & 15^{\prime} 11^{\prime \prime} & 23^{\prime} 11^{\prime \prime} & 31^{\prime} 11^{\prime \prime} & 39^{\prime} 11^{\prime \prime} & 47^{\prime} 11^{\prime \prime} \\ I & 0,858 & 0,890 & 0,873 & 0,886 & 0,880 & 0,883 \\ t & 55^{\prime} 11^{\prime \prime} & 61^{\prime} 11^{\prime \prime} & & & & \\ I & 0,839 & 0,811 & & & & \end{array}$

Vers. Nr. $1 T=25 \mathrm{sec}, c=21,2 \mathrm{mg} \mathrm{Öl} / \mathrm{m}^{3}, v=3 l / \mathrm{min}$. $t \quad 4^{\prime} 25^{\prime \prime} \quad 10^{\prime} 25^{\prime \prime} \quad 17^{\prime} 25^{\prime \prime} \quad 25^{\prime} 25^{\prime \prime} \quad 34^{\prime} 25^{\prime \prime} \quad 47^{\prime} 25^{\prime \prime}$ $\begin{array}{rrrrrrr}I & 1,62 & 2,06 & 1,98 & 1,98 & 1,96 & 1,87\end{array}$

$t 58^{\prime} 25^{\prime \prime} 63^{\prime} 25^{\prime \prime}$

I $\quad 1,87 \quad 1,85$

Vers. Nr. $5 T=40 \mathrm{sec}, c=33,8 \mathrm{mg} \mathrm{Öl} / \mathrm{m}^{3}, v=3 \mathrm{l} / \mathrm{min}$. $t 4^{\prime} 40^{\prime \prime} 12^{\prime} 40^{\prime \prime} 20^{\prime} 40^{\prime \prime} 28^{\prime} 40^{\prime \prime} 34^{\prime} 40^{\prime \prime}$

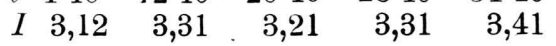

Tab. 1. Tyndallometrische Konzentrationsbestimmung der Ölnebel (Auszug aus den Versuchsprotokollen).

raumnebels in $\mathrm{mg} / \mathrm{m}^{3}$ und $v$ die Strömungsgeschwindigkeit, mit der der Gasraumnebel abge-

von 250 bzw. $375 l$ Luft enthaltene Nebelmenge auffingen. Die Nebelteilchen wurden praktisch vollständig abgefangen. Ihre Masse ergab sich aus der Gewichtszunahme der Papierfilter.

12 W. Mecklenburg, Kolloid-Z. 16, 97 [1915].

13 R. Whytlaw Gray, Smoke 1932, 127. saugt wurde. $t$ ist die Zeit in min und $I$ die relative Intensität des Streulichtes.

Die doppelt ausgeführten Versuche geben ein Bild von der guten Reproduzierbarkeit des Ölnebels. Weiterhin ist aus den Versuchsreihen abzulesen, daß die im Verlaufe eines Versuches gemessenen Streulichtintensitätswerte über eine verhältnismäßig lange Versuchsdauer hinaus konstant bleiben. In den meisten Fällen wird erst gegen Ende einer einstündigen Beobachtungsdauer eine geringe Abnahme der Streulichtintensität festgestellt. Dieselbe war stets kleiner als $10 \%$ und dürfte durch die fortlaufende Verdünnung des Gasraumnebels während der Versuche verursacht worden sein, da bei einer Absauggeschwindigkeit von $3 \mathrm{l} / \mathrm{min}$ im Verlaufe einer einstündigen Beobachtungsdauer etwa $180 l$ nebelbeladene Luft aus dem 2000-l-Gasraum entnommen werden. Die beobachtete Konstanz der Streulichtintensität wird wohl in erster Linie durch den hohen Verdünnungsgrad und die damit verbundene geringe Koagulationsgeschwindigkeit der Ölnebel verursacht. Es ist aber vielleicht auch möglich, daß die Größe der Nebelteilchen hierbei eine Rolle spielt. Aus den Untersuchungen von W. Mecklenburg ${ }^{12}$ und R. Whytlaw Gray ${ }^{13}$ über die Abhängigkeit des emittierten TyndallLichtes von der Größe der Teilchen geht nämlich hervor, daß für Aerosole von geringer Konzentration und Teilchen mit Durchmessern zwischen 1,0 bis $3,5 \times 10^{-5} \mathrm{~cm}$ die Streulichtintensität von dem Verlauf der Koagulation und dem damit verbundenen Anwachsen der Teilchen unabhängig ist, während für verdünnte Aerosole mit Teilchen, deren Durchmesser in den Bereich der Wellenlänge des sichtbaren Lichtes fallen, die Streulichtintensität während der Koagulation abnimmt.

Um ein Bild von der Größe der Apiezonöl-Nebelteilchen zu bekommen, wurde der Ölnebel unter dem Ultramikroskop beobachtet. Hierbei führen die Apiezonöl-Nebelteilchen eine so intensive $\mathrm{B}$ r o w $\mathrm{n}$ sche Bewegung aus, daß sich eine Fallbewegung nicht ausbilden kann. An Salmiaknebeln konnte dagegen die Fallgeschwindigkeit der Teilchen gemessen und daraus die Größe derselben nach der Formel von Stokes$\mathrm{Cun} n$ in $\mathrm{gh}$ a $\mathrm{m}$ berechnet werden. Hierbei ergaben sich Teilchendurchmesser zwischen 0,9 und $1,5 \times 10 \rightarrow \mathrm{cm}$. Die Apiezonöl-Nebelteilchen müssen demnach bedeutend kleiner sein als die Salmiak-Nebelteilchen.

Aus Abb. 2 geht hervor, daß für den in Frage kommenden Konzentrationsbereich die Streulichtintensität proportional der Massenkonzentration 


\begin{tabular}{|c|c|c|c|c|}
\hline $\begin{array}{l}\text { Ström.-Vol. } \\
\quad l / \mathrm{min}\end{array}$ & $T_{D}$ in ${ }^{0} \mathrm{C}$ & & & \\
\hline \multicolumn{5}{|c|}{ Versuch Nr. 41} \\
\hline $\mathbf{5}, 9$ & 23 & $\frac{I_{2^{\prime}}}{0,896}$ & $\begin{array}{c}I_{3^{\prime}} \\
0,902\end{array}$ & \\
\hline$\check{5}, 9$ & 72,5 & $\frac{I_{7^{\prime}}}{0,840}$ & $\frac{I_{8^{\prime}}}{0,840}$ & * \\
\hline 2,9 & 72,5 & $\begin{array}{c}I_{11^{\prime}} \\
0,810\end{array}$ & $\frac{I_{12^{\prime}}}{0,820}$ & \\
\hline 1 & 72,5 & $\frac{I_{18^{\prime}}}{0,786}$ & $\begin{array}{c}I_{19^{\prime}} \\
0,786\end{array}$ & \\
\hline 1 & 23 & $\frac{I_{25^{\prime}}}{0,910}$ & & \\
\hline \multicolumn{5}{|c|}{ Versuch Nr. 42} \\
\hline 5,9 & 23 & $\frac{I_{5^{\prime}}}{0,920}$ & $\frac{I_{6^{\prime}}}{0,896}$ & \\
\hline 5,9 & 72,5 & $\frac{I_{10^{\prime}}}{0,848}$ & $\frac{I_{11^{\prime}}}{0,850}$ & \\
\hline 2,9 & 72,5 & $\frac{I_{15^{\prime}}}{0,822}$ & $\frac{I_{16^{\prime}}}{0,812}$ & \\
\hline 1 & 72,5 & $\begin{array}{c}I_{24^{\prime}} \\
0,758\end{array}$ & $\frac{I_{25^{\prime}}}{0,752}$ & $\begin{array}{c}I_{26^{\prime}} \\
0,758\end{array}$ \\
\hline 1 & 23 & $\frac{I_{30^{\prime}}}{0,900}$ & 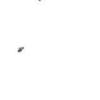 & \\
\hline \multicolumn{5}{|c|}{ Versuch Nr. 43} \\
\hline 5,9 & 23 & $\frac{I_{5^{\prime}}}{0,872}$ & $\begin{array}{c}I_{6^{\prime}} \\
0,854\end{array}$ & \\
\hline 5,9 & 167,5 & $\frac{I_{11^{\prime}}}{0, \overline{5} 78}$ & $\begin{array}{c}I_{12^{\prime}} \\
0,578\end{array}$ & \\
\hline 2,9 & 167,5 & $\frac{I_{18^{\prime}}}{0,266}$ & $\frac{I_{19^{\prime}}}{0,262}$ & \\
\hline 1 & $167, \overline{5}$ & $\frac{I_{25^{\prime}}}{0,046}$ & $\frac{I_{26^{\prime}}}{0,029}$ & $\begin{array}{c}I_{27^{\prime}} \\
0,029\end{array}$ \\
\hline 1 & 23 & $\frac{I_{31^{\prime}}}{0,910}$ & & \\
\hline \multicolumn{5}{|c|}{ Versuch Nr. 44} \\
\hline 5,9 & 23 & $\frac{I_{5^{\prime}}}{0,892}$ & $\frac{I_{6^{\prime}}}{0,910}$ & \\
\hline 5,9 & 167,5 & $\frac{I_{11^{\prime}}}{0,594}$ & $\frac{I_{12^{\prime}}}{0,590}$ & \\
\hline 2,9 & 167,5 & $\frac{I_{15^{\prime}}}{0,266}$ & $\frac{I_{16^{\prime}}}{0,266}$ & \\
\hline 1 & 167,5 & $\frac{I_{23^{\prime}}}{0,039}$ & $\begin{array}{r}I_{24^{\prime}} \\
0,028\end{array}$ & $\begin{array}{r}I_{25^{\prime}} \\
0,028\end{array}$ \\
\hline 1 & 23 & $\frac{I_{28^{\prime}}}{0,892}$ & & \\
\hline
\end{tabular}

Tab. 2. Abhängigkeit der Streulichtintensität von der Strömungsgeschwindigkeit bei konstantem Temperaturgradienten des Trennrohrs (Auszug).

\begin{tabular}{|c|c|c|c|c|c|}
\hline $\begin{array}{c}\text { Ström.-Vol. } \\
l / \text { min }\end{array}$ & $T_{D}$ in ${ }^{\circ} \mathrm{C}$ & • & & & \\
\hline \multicolumn{6}{|c|}{ Versuch Nr. 45} \\
\hline 5,9 & 23 & $\frac{I_{4^{\prime}}}{0,886}$ & $\begin{array}{c}I_{5^{\prime}} \\
0,896\end{array}$ & $\frac{I_{6^{\prime}}}{0,886}$ & \\
\hline 5,9 & 202,5 & $\frac{I_{9^{\prime}}}{0,440}$ & $\frac{I 10^{\prime}}{0,440}$ & & \\
\hline 2,9 & 202,5 & $\begin{array}{r}I_{16^{\prime}} \\
0155\end{array}$ & $I_{17^{\prime}}$ & $I_{18^{\prime}}$ & $I_{19^{\prime}}$ \\
\hline 1 & 202,5 & $\frac{I_{24^{\prime}}}{0,002}$ & 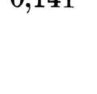 & 0,146 & 0,148 \\
\hline 1 & 23 & $\frac{I_{27^{\prime}}}{0,882}$ & & & \\
\hline
\end{tabular}

Fortsetzung von Tabelle 2.

der Ölnebel ist (Eichkurve des Tyndallometers). Bei den Intensitätswerten handelt es sich um Mittelwerte, die aus Meßreihen gemäß Tab. 1 genommen wurden.

\section{Die Abscheidungsversuche}

Versuchsreihe a: Alle Versuche dieser Reihe wurden in der Weise ausgeführt, daß im Verlaufe ein und desselben Versuches der Tempera-

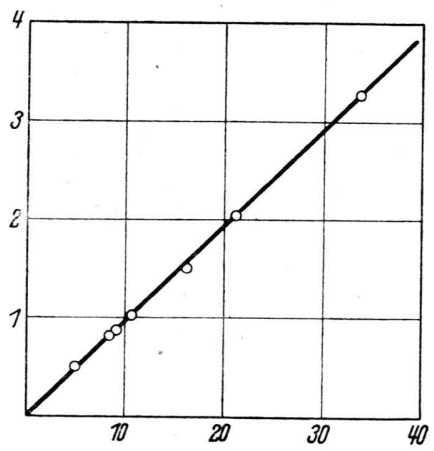

Abb. 2. Abszisse: Massenkonzentration des Gasraumnebels in $\mathrm{mg} / \mathrm{m}^{3}$.

Ordinate: Relative Streulichtintensität.

turgradient im Trennrohr konstant gehalten, die Strömungsgeschwindigkeit des Ölnebels dagegen verändert wurde. Für alle Versuche wurde die Konzentration des Gasraumnebels zu 9,3 mg $\ddot{O} \mathrm{l} / \mathrm{m}^{3}$ eingestellt. Einen Teil der Versuche zeigt Tab. 2.

Es soll am Versuch Nr.45, Tab.2, als Beispiel beschrieben werden, wie derselbe im einzelnen ausgeführt wurde (vergl. hierzu auch Abb.1): Nachdem die Konzentration des Gasraumnebels in 
beschriebener Weise eingestellt worden war, wurde derselbe nach Öffnen des Verschlusses $V_{1}$ durch das Trennrohr und Tyndallometer abgesaugt (Ström.-Vol. 5,9 l/min) und die Aufhellung im Tyndallometer nach der vierten, fünften und sechsten Minute, vom Beginn des Einleitens ab gerechnet, abgelesen. Auf Grund der drei erhaltenen Intensitätswerte von $0,886,0,896$ und 0,886 konnte die Streulichtintensität als konstant bleibend angesehen werden. Die Intensität des gestreuten Lichtes war bei isothermem Trennrohr von dem Strömungsvolumen des Ölnebels in dem in Frage kommenden Bereich von $1 l /$ min bis $6 \mathrm{l} / \mathrm{min}$ unabhängig. Nun wurde der Heizdraht auf Temperatur gebracht $\left(T_{D}=202,5^{\circ} \mathrm{C}\right)$ und

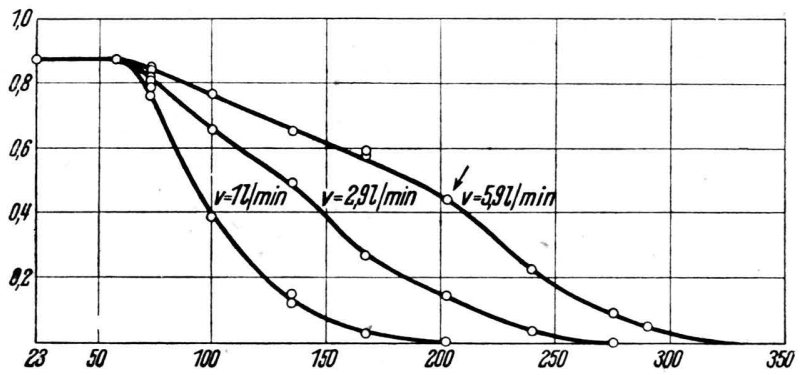

Abb. 3. Abszisse: Heizdrahttemperatur in ${ }^{\circ} \mathrm{C}$. Ordinate: Nebeldurchlaß des Trennrohrs; aufgetragen ist die Streulichtintensität.

nach der neunten und zehnten Minute bei gleichbleibendem Strömungsvolumen der nebelbeladenen Luft von 5,9 l/min abermals die Tyndallometeraufhellung festgestellt. Die abgelesenen Intensitätswerte waren um einen erheblichen Betrag geringer als die gemessenen Werte bei stromlosem Trennrohr. Sie stimmten jedoch gut miteinander überein, ein Zeichen dafür, daß die Teilchenkonzentration des das Trennrohr verlassenden Nebels einen konstanten Wert angenommen hatte. Daraufhin wurde die Absaugegeschwindigkeit des Ölnebels auf 2,9 $\mathrm{l} / \mathrm{min}$ erniedrigt. Abermals nahm die Streulichtintensität beträchtlich ab und näherte sich am Ende der 18. und 19. Minute dem konstanten $I$-Wert von 0,148 . Weitere in gleicher Weise ausgeführte Messungen bei einem Strömungsvolumen von $1 \mathrm{l} / \mathrm{min}$ vervollständigten den Versuch. Zum Schluß wurde dann der Nebelstrom bei stromlosem Trennrohr nochmals auf Konstanz geprüft und meistenteils die zu Anfang gemessenen Intensitätswerte bestätigt gefunden (vergl. Vers. Nr. $45 T_{D}=23^{\circ} \mathrm{C}$; Ström.-Vol. $\left.=1 \mathrm{l} / \mathrm{min}\right)$.
Die doppelt ausgeführten Versuche sollen zeigen, wie ausgezeichnet sich die einzelnen Messungen reproduzieren lassen. Das Ergebnis der Versuche dieser Reihe zeigt Abb. 3.

Aus Abb. 3 ist zu entnehmen, daß für alle Strömungsvolumina die Abscheidung der Nebelteilchen erst oberhalb einer Heizdrahttemperatur von $60^{\circ} \mathrm{C}$ einsetzt. Eine vollständige Abscheidung der strömenden Ölnebel gelingt für die untersuchten Strömungsvolumina von $1,2,9$ und $5,9 \mathrm{l} / \mathrm{min}$ bei Heizdrahttemperaturen von 205,275 und $325^{\circ} \mathrm{C}$. Im Falle der höheren Heizdrahttemperaturen werden Nebelteilchen, die in die Nähe des Heizdrahtes

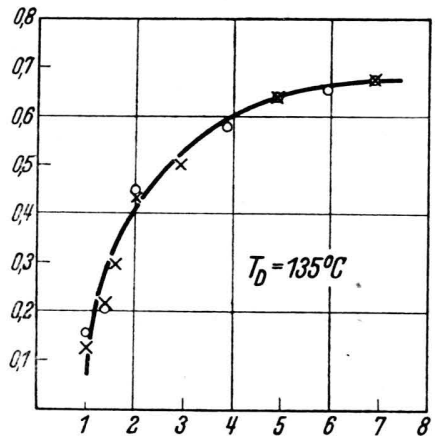

Abł. 4. Abszisse: Strömungsvolumen in $l / \mathrm{min}$. . Ordinate: Nebeldurchlaß des Trennrohrs; aufgetragen ist die Streulichtintensität.

$\odot$ Versuch Nr. 38; X Versuch Nr. 39.

gelangen, verdampfen. Hierbei dürfte eine turbulente Strömung den Vorgang des Verdampfens insofern begünstigen, da hierdurch mehr Teilchen in die Nähe des Heizdrahtes gelangen werden als bei laminarer Strömung. Vielleicht ist der Knick in der Abscheidungskurve des mit einer Geschwindigkeit von $5,9 \mathrm{l} / \mathrm{min}$ strömenden Nebels auf solche Einflïsse zurückzuführen (vergl. den Pfeil in Abb. 3).

Bei den Versuchen Nr. 37 und 38 wurden auch noch andere Strömungsvolumina berücksichtigt und die Abscheidung gemessen. Als Ergebnis der beiden Versuche wurde die Kurve in Abb. 4 erhalten, welche den Einfluß der Strömungsgeschwindigkeit auf die. Abscheidung wiedergibt. Höchstwahrscheinlich wird bei den Strömungsvolumina $>1,5 \mathrm{l} / \mathrm{min}$ die Abscheidung auch von der Turbulenz der Strömung beeinflußt.

Versuchsreihe b: Anschließend werden Versuche mitgeteilt, bei denen im Verlaufe ein und desselben Versuches das Strömungsvolumen konstant gehalten und der Temperaturgradient fort- 
laufend verändert wurde. Es schien in diesem Falle nicht ratsam, den gesamten Kurvenzug durch einen Versuch vollkommen erfassen zu wollen, da solche Versuche zu lange Zeit in Anspruch genommen und die in dieser Zeit an den Nebelsystemen eingetretenen Veränderungen die Meßergebnisse in größerem Maße beeinflußt hätten. Deshalb wurde das Kurvenbild etappenweise erhalten. Die Untersuchungen erstrecken sich auf zwei Nebel mit verschiedenen Ausgangskonzentrationen. Die einzelnen Versuche, alle bei einem Strömungsvolumen der nebelbeladenen Luft von $1 \mathrm{l} / \mathrm{min}$ ausgeführt, sind in Abb. 5 zusammengefaßt. Hierbei sind die zusammengehörenden Meßpunkte eines Versuches als solche gekennzeichnet.

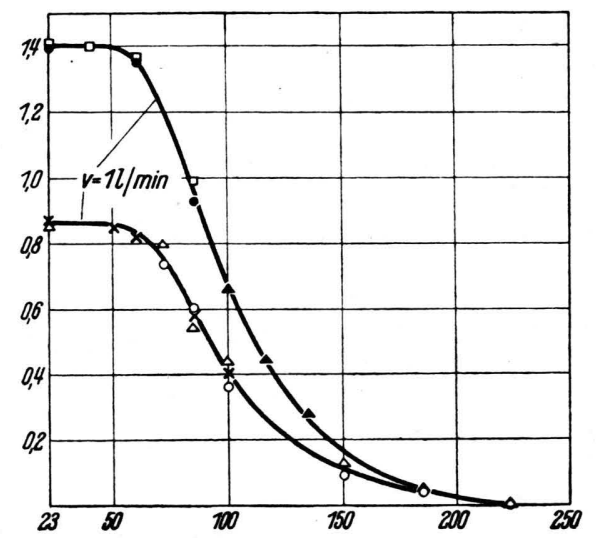

Abb. 5. Abszisse: Heizdrahttemperatur in ${ }^{\circ} \mathrm{C}$. Ordinate: Nebeldurchlaß des Trennrohrs; aufgetragen ist die Streulichtintensität.

Die Abscheidungskurve für den Nebel mit der kleineren Massenkonzentration in Abb. 5 entspricht in ihrem Verlauf ganz der in Abb. 3 bei einem Strömungsvolumen von $1 \mathrm{l} / \mathrm{min}$ festgestellten. Im Zusammenhang mit der Abscheidungskurve für den konzentrierteren Nebel folgt, daß die Abscheidung proportional der Konzentration der Teilchen ist.

In Verbindung mit der Bestimmung der Heizdrahttemperaturen wurden auch Temperaturmessungen bei luftdurchströmtem Trennrohr in verschiedener Höhe des Heizdrahtes mit Hilfe eines Thermoelementes ausgeführt. Die festgestellten Temperaturen sind dort wegen der Luftdurchströmung verschieden und hängen weiterhin von der eingestellten Strömungsgeschwindigkeit ab. Hierdurch werden Abweichungen von den angegebenen Temperaturwerten bedingt, da während deren Bestimmung das Trennrohr nicht von Luft durchströmt wurde. Bei Luftdurchströmung werden meistens etwas höhere Temperaturen beobachtet, die bei den höheren Heizdrahttemperaturen in einigen Fällen bis $\mathrm{zu} 12^{\circ} \mathrm{C}$ über den angegebenen Werten liegen können.

Was die Temperaturverteilung im Trennrohr anbelangt, so liegen im vorliegenden Falle wegen der dauernden Luftdurchströmung keine einfachen Verhältnisse vor. Angaben über die Temperaturverteilung im Trennrohr finden sich bei Clusius und Dickel ${ }^{14}$.

IV. Die im thermischen Felde auf Schwebeteilchen ausgeübten Kraftwirkungen unter Berücksichtigung der Versuchsresultate

Über die durch einen Temperaturgradienten hervorgerufene Kraft an einem Teilchen, das gegenüber der mittleren freien Weglänge als klein angesehen werden kann, geben Berechnungen von A. Einstein Auskunft ${ }^{3}$. Die Kraft wird dadurch verursacht, daß die in Richtung des Gefälles auf die Oberfläche der Teilchen auftreffenden Gasmoleküle einen größeren Impuls übertragen als die in entgegengesetzter Richtung auftreffenden Moleküle. So resultiert eine Geschwindigkeit $v$ der Teilchen in Richtung des Gefälles. Nach A. Einstein ist

$$
v=-\frac{1}{8} c \frac{\lambda}{T} \frac{\partial T}{\partial x} ;
$$

In dieser Gleichung bedeutet $\lambda$ die mittlere freie Weglänge, $c$ die mittlere Geschwindigkeit der Gasmoleküle, $T$ die absolute Temperatur und $x$ die Achse, längs der ein Temperaturgefälle herrscht.

Obwohl Gl. (1) nur auf Teilchen $<10^{-5} \mathrm{~cm}$ angewandt werden kann, erschien es interessant genug, Berechnungen mit Hilfe derselben an Hand der Versuchsresultate vorzunehmen. Hierzu wurde aus der Verweilzeit der Teilchen im Trennrohr und dem Umstand, daß die Teilchen, die sich in nächster Nähe des Heizdrahtes befinden, einen Weg von etwa $6,5 \mathrm{~mm}$ zurückzulegen haben, um auf der Glasrohrwand abgeschieden zu werden, die horizontale Geschwindigkeitskomponente ermittelt. In Tab. 3 finden sich für die verschiedenen untersuchten Strömungsvolumina die zugehörigen Werte für die Verweilzeiten und die horizontalen Geschwindigkeitskomponenten verzeichnet (vergl. Spalte I, II und IV). Da nun durch die Versuche diejenigen Temperaturgradienten bekannt sind, bei denen eine vollständige Abscheidung der Teilchen eintrat (vergl. Abb. 3), so

14 K. Clusius u. O. Dickel, Z. physik. Chem. Abt. B 44, 412 [1939]. 


\begin{tabular}{|c|c|c|c|c|}
\hline $\begin{array}{l}\text { I. } \\
\text { Ström.- } \\
\text { Vol. in } \\
l / \mathrm{min} .\end{array}$ & \begin{tabular}{|c|} 
II. \\
Ver- \\
weil- \\
zeit in \\
sec
\end{tabular} & $\begin{array}{c}\text { III. } \\
\text { Temperatur- } \\
\text { gradient zur } \\
\text { vollständigen } \\
\text { Abscheidung } \\
\text { in }{ }^{\circ} \mathrm{C} / \mathrm{cm}^{*}\end{array}$ & $\begin{array}{c}\text { IV. } \\
\text { horizontale } \\
\text { Geschwin- } \\
\text { digkeits- } \\
\text { komponente } \\
\text { in } \mathrm{mm} / \mathrm{sec}\end{array}$ & $\begin{array}{l}\text { V. } \\
\text { Geschwindig- } \\
\text { keit der Teil- } \\
\text { chen, nach Gl. } \\
\text { (1) berechnet } \\
\text { in } \mathrm{mm} / \mathrm{sec}\end{array}$ \\
\hline $\begin{array}{l}1,0 \\
2,9 \\
5,9\end{array}$ & $\begin{array}{l}7,5 \\
2,6 \\
1,3\end{array}$ & $\begin{array}{l}315 \\
423 \\
500\end{array}$ & $\begin{array}{l}0,9 \\
2,5 \\
5,0\end{array}$ & $\begin{array}{l}1,3 \\
1,8 \\
2,1\end{array}$ \\
\hline
\end{tabular}

Tab. 3. Vergleich der aus dem Abscheidungserfolg berechneten Teilchengeschwindigkeit mit der aus dem

Temperaturgradienten nach Gl. (1) berechneten Teilchengeschwindigkeit.

wurde mit Hilfe von Gl. (1) die Geschwindigkeit der Teilchen bei diesen Gradienten berechnet. In

* Es wurde angenommen, daß der gesamte Temperaturabfall zwischen Heizdraht und Glasrohrwand erfolgt.
Spalte III sind für die verschiedenen Strömungsvolumina die Werte der in Frage kommenden Temperaturgradienten angegeben. In Spalte V finden sich schließlich die bei diesen Gradienten mit Hilfe von Gl. (1) errechneten Geschwindigkeitswerte der Teilchen verzeichnet.

Vergleicht man die in Spalte IV und V einander entsprechenden Geschwindigkeitswerte, so zeigen diese bei den Strömungsvolumina von 1 und 2,9 l/min verhältnismäßig gute Übereinstimmung, während für die höhere Strömungsgeschwindigkeit von $5,9 \mathrm{l} / \mathrm{min}$ etwas auseinanderliegende Werte erhalten werden. Da Gl. (1) eigentlich nur für ruhende Systeme gilt, sich bei immer länger werdender Verweilzeit der Teilchen im Trennrohr die Verhältnisse diesem Zustand aber immer mehr nähern, so verdienen insbesondere die für lange Verweilzeiten erhaltenen Werte Beachtung.

\title{
Widerstandsnormale bei hohen Frequenzen
}

\author{
Von Hans Heinrich Meinke
}

(Z. Naturforschg. 2 a, 55-59 [1947]; aus Hamburg eingegangen am 3. Juli 1946)

\begin{abstract}
Als Widerstandsnormal wird der Wellenwiderstand einer Leitung benutzt, der in einigen Fällen sehr genau berechnet und mit Genauigkeiten bis zu etwa 2\%o praktisch dargestellt werden kann. Ein Meßverfahren zum Abgleich nicht berechenbarer Wellenwiderstände wird angegeben.
\end{abstract}

$D^{i}$ ie Einheit des Widerstandes ist bei Gleichstrom definiert. Ihre Übertragung auf Wechselströme stößt mit wachsender Frequenz auf immer größere Schwierigkeiten. Bei hohen Frequenzen $\left(f>10^{7} \mathrm{~Hz}\right)$ mißt man einen Widerstand in bekannter Weise dadurch, daß man ihn als Abschlußwiderstand hinter eine homogene Leitung setzt (Abb. 1) und den Verlauf des Absolutwertes des Stromes oder der Spannung längs dieser Leitung mißt. Der gemessenen Kurve, z. B. der Spannungskurve, entnimmt man den Maximalwert $U_{\max }$ und den Minimalwert $U_{\min }$ sowie den Abstand $l$ des Spannungsminimums vom Meßobjekt (Abb.1). Bei Vernachlässigung der Leitungsdämpfung ist der gesuchte Widerstand in komplexer Darstellung

$$
\mathfrak{X}=3 \frac{\frac{U_{\min }}{U_{\max }}-j \operatorname{tg} 2 \pi \frac{l}{\lambda}}{1-j \frac{U_{\min }}{U_{\max }} \operatorname{tg} 2 \pi \frac{l}{\lambda}},
$$

wobei 3 der komplexe Wellenwiderstand der Leitung ist. Das Meßverfahren selbst ergibt also mit Hilfe der Größen $U_{\min } / U_{\max }$ und $l / \lambda$ lediglich den

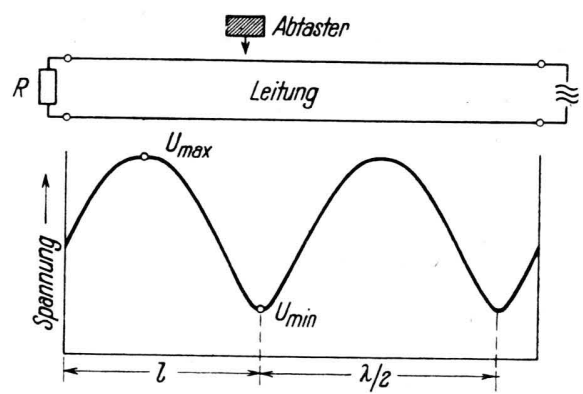

Abb. 1. Meßverfahren und Spannungskurve.

Quotienten $\Re / 3$. Man erkennt an der Formel, daß der Wellenwiderstand 3 bei diesem Meßverfahren die Rolle des Normalwiderstandes übernimmt, mit dem der unbekannte Widerstand $\Re$ verglichen wird. Im folgenden wird nun gezeigt, wie man 